\title{
The differential impact of volatile and intravenous anaesthetics in humans
}

\author{
Nicholas J. Sarlis ${ }^{1}$, Christian A. Koch ${ }^{2}$ \\ ${ }^{1}$ Department of Medical Affairs - Oncology, Sanofi-Aventis U.S., Bridgewater, $N J$, ${ }^{2}$ Division of Endocrinology, University \\ of Mississippi Medical Center, Jackson, MS, USA
}

Re.: Kostopanagiotou G, et al. 2010 The differential impact of volatile and intravenous anaesthetics on stress response in the swine. Hormones 9: 67-75

\section{Dear Editor,}

We read with interest the contribution by Dr. Kostopanagiotou and colleagues describing the differential impact of volatile and intravenous anaesthetics on select hormonal and immune parameters of the stress response in the swine. ${ }^{1}$ We would like to highlight earlier human data of ours (some of which unpublished to date) as well as from others that are pertinent to this interesting line of research and generally supportive of the authors' conclusions.

One of us (NJS) participated in a pilot clinical study involving 55 healthy postmenopausal women who had undergone total intravenous anaesthesia (TIVA) with various agents in the context of elective surgery for non-infectious or non-trauma indications in order to investigate their hypothalamic-pituitary (HP) axis responses to such agents. ${ }^{2}$ The anaesthetics

Key words: Anaesthetics, Fentanyl, HPA axis, Ketamin, Stress response

\section{Address for correspondence:}

Prof. Dr. Med. Habil. Christian A. Koch, FACP, FACE, Division of Endocrinology, University of Mississippi Medical Center, 2500 North State Street, University of Mississippi Medical Center, Jackson, MS 39216, USA, Phone: +1-601-984-5495, Fax: +1-601-984-5769, E-mail: ckoch@umc.edu

Received 25-03-10, Revised 20-05-10, Accepted 10-06-10 used (in various combinations and at standard doses) were: thiopental, ketamine, dehydrobenzperidol (droperidol), fentanyl, diazepam, and midazolam. Pancuronium or vecuronium was used briefly only during intubation, and atropine was given solely on an as-needed basis. No volatile anaesthetics were used, while standard monitoring of cardiovascular, hemodynamic or respiratory parameters took place, although was not formally recorded for data analysis in this clinical study. Sampling and hormonal investigations in all patients were performed: a. within 15 min prior to entering the anaesthesia suite ("true" baseline), b. at the time of initiation of anaesthesia induction, and c. during the period between 5 min since completion of the induction phase of anaesthesia and entry into a body/articular cavity or superficial organ (e.g. breast or lymph nodes). The skin/subcutis/fascial incision time was included in the latter period. The depth of anaesthesia was monitored via the Patient Response to Surgical Stimulus (PRST) score. ${ }^{3}$ Blood samples were obtained every $5 \mathrm{~min}$ for a period of $18.5+$ 12.3 min (mean $+\mathrm{SD}$ ) following completion of the induction phase of anaesthesia. Trends were analyzed using standard statistical methods and all parameters were compared to baseline. The following variables were measured in either plasma or serum: Prolactin (PRL), Thyrotropin (TSH), Free Thyroxine (FT4), Total Triiodothyronine (TT3), Adrenocorticotropin hormone (ACTH), Cortisol (F), $\beta$-Endorphin ( $\beta$-EP), 
$\beta$-Lipotropin ( $\beta$-LPH), Corticotropin-Like Intermediate Peptide (CLIP), Epinephrine (E), Norepinephrine (NE), Dopamine (DA), Neuropeptide Y (NPY), Interleukin-1 $\beta$ (IL-1 $\beta$ ), IL-6, and circulating soluble IL-2 receptor alpha (sIL2R $\alpha)$.

The results of our earlier analysis for PRL and the HP-thyroid axis parameters have been previously published. ${ }^{2}$ These showed that thiopental and ketamine (probably via a cholinergic mechanism), as well as dehydrobenzperidol (droperidol) (most likely via a dopaminergic mechanism), increased PRL levels significantly $(\mathrm{p}<0.05)$, while fentanyl, diazepam, and midazolam did not alter basal PRL values. TSH, $\mathrm{FT}_{4}$, and TT3 levels remained unaffected by all drugs tested, though ketamine, a $N$-methyl-D-aspartate (NMDA) receptor antagonist, showed a non-statistically significant tendency to increase both TSH and TT3 levels ( $p$ values: $0.08-0.10$ ). The relative lack of response of TSH values to the stress of intravenous anaesthesia and/or cavity non-breaching surgical incision was attributed to either the slower release of TSH stores or the possible involvement of a central somatostatinergic mechanism. ${ }^{2}$

We now proceeded with re-analysis of the remainder of the earlier data (which had remained unreported). In agreement with the findings by Kostopanagiotou et al, as well as others, ${ }^{4-7}$ significant and persistent increases were found in $\mathrm{ACTH}, \mathrm{F}$, $\beta$-EP, $\beta$-LPH, and CLIP for all drugs $(\mathrm{p}<0.05)$ except diazepam and midazolam. The highest degree of activation of the HP-adrenal axis was seen with ketamine and fentanyl. Of interest, this is in contrast to findings in rats exposed to 40 days of chronic mild stress, in which circulating ACTH and corticosterone levels were normalized by acute and chronic ketamine treatment. ${ }^{8}$ Plasma DA, IL-1 $\beta$, and IL-6 did not change over time in any of the patients, irrespective of TIVA agent administered, with the exception of a mild upward trend in DA levels following thiopental-which remained statistically non-significant vs. baseline. Levels of circulating SIL-2R $\alpha$ remained fairly low in all patients, with the exception of one patient in whom a high baseline value decreased post-midazolam. This pattern of response of inflammatory markers was overall consistent with the report by Kostopanagiotou et al; however, others have observed significant increases in plasma cytokines, especially IL-6, during anesthesia. ${ }^{9}$ A complex pattern of mostly parallel changes in E, NE, and NPY were observed, most consistent with a biphasic response to thiopental and ketamine, with a pattern showing an intermediate peak and then a smooth decrease in levels. No effect on catecholamine output was seen with droperidol, diazepam or midazolam, while a statistically significant decrease in NE (but not $\mathrm{E}$ or NPY) levels was observed after fentanyl. We could not exclude the possible interference of cardiovascular instability with the catecholamine level changes. With regard to catecholamine excursions, under sevoflurane-based anaesthesia, patients who received intermittent boluses of fentanyl had higher levels of $\mathrm{NE}$ and E 30 min after starting cardiopulmonary bypass than those receiving remifentanil. ${ }^{7}$ Moreover, in children undergoing inguinal herniotomy, plasma E levels were significantly reduced in those who were given intravenous fentanyl compared to the control group who had general anaesthesia plus a caudal block, while plasma NE levels were not different between these groups. ${ }^{10}$

In conclusion, our data in humans strongly support the hypothesis brought forth by Kostopanagiotou et al, i.e. that various general anaesthetics exert a differential effect on the hormonal/immune stress response (independently of the effect of the surgical insult per se). Further, we also concur with the notion that one would ideally need to choose a particular type of anaesthetic in order to obtain a preferred pattern of reaction of the stress system, which would be optimally homeostatic and befitting to the particular clinical situation. Although data on this topic are rapidly accumulating ${ }^{11,12}$ more extensive clinical studies are needed. Indeed, the matching of specific anaesthetic agents to specific clinical scenarios-based on neurohumoral, hormonal, and immune data-could lead to substantial improvement in patient outcomes both in surgery and in intensive care settings.

\section{DISCLOSURES}

Dr. Nicholas J. Sarlis is an employee of sanofiaventis U.S. and holds stock options, as well as stock, in this company. However, no real or perceived conflict of interest exists with regard to this communication. Dr. Christian A. Koch has served as consultant for 
Novo Nordisk, is principal investigator of the Corlux and the TR321 trials, and has nothing relevant to disclose.

\section{REFERENCES}

1. Kostopanagiotou G, Kalimeris K, Christodoulaki, et al, C 2010 The differential impact of volatile and intravenous anaesthetics on stress response in the swine. Hormones (Athens) 9: 67-75.

2. Kaniaris PK, Sarlis NJ, Satti A, 1989 Hypothalamohypophyseal response to drugs used in anesthesia. Middle East J Anesthesiol 10: 195-209 (Erratum in: Middle East J Anesthesiol 10: 333).

3. Evans JM, Davies WL, 1984 Monitoring anaesthesia. Clin Anesth 2: 243-262.

4. Brandt MR, Korshin J, Hansen AP, et al, 1978 Influence of morphine anaesthesia on the endocrine-metabolic response to open-heart surgery. Acta Anaesthesiol Scand 22: 400-412.

5. Nilsson A, Persson MP, Hartvig P, Wide L, 1988 Effect of total intravenous anaesthesia with midazolam/alfentanil on the adrenocortical and hyperglycaemic response to abdominal surgery. Acta Anaesthesiol Scand 32: 379382.

6. Crozier TA, Sumpf E, 1996 The effect of total intravenous anesthesia with $\mathrm{S}-(+)$-ketamine/propofol on hemodynamic, endocrine and metabolic stress reactions in comparison to alfentanil/propofol in laparotomy. Anaesthesist 45: 1015-1023.

7. Winterhalter M, Brandl K, Rahe-Meyer N, et al, 2008 Endocrine stress response and inflammatory activation during $\mathrm{CABG}$ surgery. A randomized trial comparing remifentanil infusion to intermittent fentanyl. Eur $\mathbf{J}$ Anaesthesiol 25: 326-335.

8. Garcia LS, Comim CM, Valvassori SS, et al, 2009 Ketamine treatment reverses behavioral and physiological alterations induced by chronic mild stress in rats. Prog Neuropsychopharmacol Biol Psychiatry 33: 450-455.

9. Crozier TA, Müller JE, Quittkat D, Sydow M, Wuttke W, Kettler D, 1994 Effect of anaesthesia on the cytokine responses to abdominal surgery. Br J Anaesth 72: 280285.

10. Somri M, Tome R, Teszler CM, et al, 2007 Does adding intravenous fentanyl to caudal block in children enhance the efficacy of multimodal analgesia as reflected in the plasma level of catecholamines? Eur J Anaesthesiol 24: 408-413.

11. Shorrab AA, Atallah MM, 2003 Total intravenous anaesthesia with ketamine-midazolam versus halothane-nitrous oxide-oxygen anaesthesia for prolonged abdominal surgery. Eur J Anaesthesiol 20: 925-931.

12. Mistraletti G, Donatelli F, Carli F, 2005 Metabolic and endocrine effects of sedative agents. Curr Opin Crit Care 11: 312-317.

Grants or fellowship supports: The initial study whereupon this letter is based was supported in part by F. Hoffmann-La Roche Ltd., Basel, Switzerland, and Janssen Pharmaceutica N.V., Beerse, Belgium.

Other disclosures: Dr. Nicholas J. Sarlis is an employee of sanofi-aventis U.S. and holds stock options, as well as stock, in this company. However, no real or perceived conflict of interest exists with regard to this communication. Dr. Christian A. Koch has served as consultant for Novo Nordisk, is principal investigator of the Corlux and the TR321 trials, and has nothing relevant to disclose. 